\title{
The role of peri-traumatic stress and disruption distress in predicting post-traumatic stress disorder symptoms following exposure to a natural disaster
}

Joseph M. Boden, David M. Fergusson, L. John Horwood and Roger T. Mulder

\section{Background}

Few studies have examined the contribution of specific disaster-related experiences to post-traumatic stress disorder (PTSD) symptoms.

\section{Aims}

To examine the roles of peri-traumatic stress and distress due to lingering disaster-related disruption in explaining linkages between disaster exposure and PTSD symptoms among a cohort exposed to the 2010-2011 Canterbury (New Zealand) earthquakes.

\section{Method}

Structural equation models were fitted to data obtained from the Christchurch Health and Development Study at age 35 ( $n=495), 20-24$ months following the onset of the disaster. Measures included: earthquake exposure, peri-traumatic stress, disruption distress and PTSD symptoms.

\section{Results}

The associations between earthquake exposure and PTSD symptoms were explained largely by the experience of peritraumatic stress during the earthquakes $(\beta=0.189, P<0.0001)$ and disruption distress following the earthquakes ( $\beta=0.105$, $P<0.0001)$.

\section{Conclusions}

The results suggest the importance of minimising post-event disruption distress following exposure to a natural disaster.

\section{Declaration of interest}

None.

\section{Copyright and usage}

(c) The Royal College of Psychiatrists 2015. This is an open access article distributed under the terms of the Creative Commons Non-Commercial, No Derivatives (CC BY-NC-ND) licence.
In recent years, there has been significant interest in the mental health and psychosocial impacts of exposure to natural disasters, ${ }^{1-5}$ with a particular focus on post-traumatic stress disorder (PTSD). ${ }^{3,4,6,7}$ A number of studies have shown elevated rates of PTSD symptomatology among individuals exposed to natural disasters, with those exposed to higher levels of potentially lifethreatening events being at the greatest risk for developing PTSD symptomatology. ${ }^{8-11}$

One question arising from this research is the extent to which features of a disaster ${ }^{12,13}$ may explain elevated rates of PTSD symptomatology among those exposed. ${ }^{14}$ Specifically, two general aspects of natural disasters may influence PTSD symptomatology. First, PTSD symptomatology may be a response to the immediate features of the disaster. In the case of earthquakes, these would include the experience of the violent shaking of the ground and structures and witnessing damage to buildings or infrastructure as it occurs. The view that PTSD is primarily related to these experiences is supported by the neuropsychological literature on PTSD, which suggests that the disorder is strongly related to the experience of fear, with the neural circuitry implicated in PTSD being involved in the regulation of the fear response. $^{15-17}$

Second, there is growing evidence that stressful and disruptive environmental and personal conditions that exist following a disaster may also lead to increased PTSD symptoms. For example, Newnham et $a l^{18}$ found that displacement and other stressful events during a civil war were associated with increased risks of PTSD, in addition to exposure to actual fighting. Similarly, Parslow et al, ${ }^{19}$ using data from a sample of individuals exposed to bushfires, reported that PTSD was linked to both exposure to the fires and also to the experience of evacuation and displacement. Also, Tracy and colleagues ${ }^{20}$ found that increased rates of PTSD were related not only to hurricane exposure but also to loss or damage caused by the hurricanes. In the case of earthquakes, the stressful and disruptive factors (hereafter referred to as disruption distress) following the events may include damage to one's home, disruption to routines of daily living, difficulty in maintaining employment and related factors that create high levels of stress on a day-to-day basis.

In this paper, we address this issue by examining the relative contributions of peri-traumatic stress and disruption distress on the PTSD responses of a birth cohort, the Christchurch Health and Development Study (CHDS). Beginning in September 2010, the City of Christchurch in New Zealand and the surrounding Canterbury region were struck by a series of over 10000 earthquakes, with four major earthquakes causing widespread property damage to the city, and one (22 February 2011) resulting in 185 deaths. The city of Christchurch is also home to the longrunning CHDS, a birth cohort of 1265 children born in 1977. Of this cohort, more than $50 \%$ were exposed to the Canterbury earthquakes, and at age 35 those exposed were interviewed about their experiences of these earthquakes. The purpose of the present investigation was to examine the linkages between earthquake exposure and PTSD symptoms, comparing the roles played by: (a) peri-traumatic stress as a result of the earthquakes; and (b) disruption distress in the aftermath of the earthquakes.

The aims of this analysis were to fit a structural equation model estimating the relative contribution of peri-traumatic stress and disruption distress in explaining the association between earthquake exposure and the development of PTSD symptoms. Details of model fitting and model specifications are provided in the Method. Using structural equation modelling (SEM), it proves possible to estimate the direct and indirect pathways in the model 
and to compare the fractions of the correlation between earthquake exposure and PTSD symptoms that are explained by each pathway in the model.

\section{Method}

\section{Participants}

The data were collected at the age 35 assessment of the CHDS, a longitudinal study of a cohort of 1265 children born in the Christchurch (New Zealand) urban region over a 4-month period from April to August 1977. The cohort comprised 98\% of all children born in the Christchurch region during this period. This cohort has now been studied from birth to age 35 years. ${ }^{21,22}$ All aspects of data collection have received ethical approval by the Canterbury regional health and disability ethics committee, and all data were collected with the explicit consent of study participants.

\section{Data collection}

In 2012, members of the CHDS cohort were approached to respond to the age 35 assessment for the study. This process identified 962 cohort members available for contact, who represented $79 \%$ of the surviving cohort. Of these, 505 were resident in Canterbury during the earthquakes. These cohort members were invited to participate in a further interview concerning their exposure to the Canterbury earthquakes. Of those eligible, 495 respondents completed an interview of approximately $1 \mathrm{~h}$ that assessed their earthquake experiences and reactions to the earthquakes. These interviews took place approximately 20-24 months following the start of the Canterbury earthquakes in September 2010. By the time the interviews began, cohort members could have been exposed to four major earthquakes ranging in Richter Scale magnitude from 6.0 to 7.1. These earthquakes occurred on 4 September 2010, 22 February 2011, 13 June 2011 and 23 December 2011. Interviews took place during the period May-October 2012.

\section{Extent of earthquake exposure}

For each of the four major earthquakes, participants were asked a series of questions relating to the severity and immediate impact of the earthquakes. Items were based on the Modified Mercalli Earthquake Intensity $\mathrm{Scale}^{23}$ and designed to examine the consequences of the earthquakes for property, buildings, land and infrastructure. Using this information a series of 11 indices were constructed to reflect the severity of the four major earthquakes. These indices were scaled on a 3-point scale ranging from $0=$ no/minor impact, $1=$ mild $/$ moderate impact and $2=$ severe $/$ major impact. Individuals not exposed to a specific earthquake were coded as zero on all indices for that earthquake. To reduce the dimensionality of the data, for each index scores were summed across the four earthquakes to produce a total score. These scores were then used in a confirmatory factor model to test for unidimensionality. ${ }^{24}$ This analysis showed that, with some allowance for correlated item specificity, the scale items fitted a single-factor model representing the severity of the individual's earthquake exposure (details of the development of this measure are available in Fergusson et $a l^{24}$ ). The scale was found to have good reliability $(\alpha=0.92)$. The mean score for the present sample was 32.91 (s.d.=14.66; range $0-84.0$ ).

\section{Earthquake-specific PTSD symptoms}

Earthquake-related PTSD symptomatology was assessed using the Diagnostic Interview Schedule. ${ }^{25}$ Participants were questioned regarding 15 symptoms arising specifically from the Canterbury earthquakes. These symptoms included: re-experiencing the disaster, emotional or physical reactions to remembering the earthquakes, avoidance, lack of memory for events during the earthquakes, dissociation and anxiety/increased arousal. Each item was answered using a 3-point scale for each symptom with the following answer options: $1=$ 'doesn't apply', $2=$ =applies somewhat' and $3=$ 'definitely applies' (those answering ' 3 ' were coded as having the symptom). The questions were asked for symptoms that had occurred at any time since the earthquakes. The measure of the extent of PTSD symptoms was created for each participant by summing the scores for the 15 items. This scale was found to have good reliability $(\alpha=0.92)$. The mean score for the present sample was 1.37 (s.d.=2.70; range $0-15$ ).

In addition, for the purposes of supplementary analyses, participants were classified as meeting criteria for a DSM-IV ${ }^{26}$ diagnosis of PTSD if they reported experiencing the following symptom pattern during the period following exposure to the earthquakes: at least one symptom of re-experiencing the disaster; at least three symptoms related to avoidance, memory issues or dissociation; and at least two symptoms related to anxiety/ increased arousal. This information was used to create a threelevel classification measure in which: 1='no PTSD symptoms'; $2=$ 'reported one or more PTSD symptoms but did not meet criteria for PTSD' (21.8\% of respondents); and 3='met criteria for PTSD diagnosis' (3.2\% of respondents).

\section{Peri-traumatic stress}

Peri-traumatic stress was assessed via the Peritraumatic Distress Inventory (PDI), ${ }^{27}$ which was supplemented by three customwritten survey items relating to: fear/panic and dissociative symptomatology. The PDI is a 13 -item scale designed to assess the distress experienced during and immediately after exposure to a critical event such as a natural disaster. Questions were asked using a 5-point Likert scale response format ranging from $1=$ 'not at all' to $5=$ 'a great deal', regarding peri-traumatic stress experienced during any of the four Canterbury earthquakes of magnitude 6.0 or greater. The scale included such items as: 'felt afraid for your own safety', 'felt horrified by what you saw' and 'thought you might die'. A scale score was derived for each participant by summing the 13 items and the 3 additional items and dividing by the number of items. The scale was found to have good reliability $(\alpha=0.89)$. The mean score for the present sample was 2.02 (s.d.=0.71; range $1-4.5$ ).

\section{Disruption distress}

Participants were also asked a series of 12 questions regarding the extent to which the earthquakes had caused distress related to disruption of their living situation, activities and daily routines. These questions assessed the level of distress caused by: having to move house or leave the area; disruption of family life, children's schooling and uncertainty about the future; disruption of services (power; water; sewerage); disruption to employment; loss of community; family and friends moving house or leaving the area; uncertainty about the continuing aftershocks; and difficulties in getting answers about property status or repair/ rebuilding of the family home. ${ }^{24,28}$

In all cases, items were scored on a 5-point scale ranging from $1=$ 'not at all' to $5=$ 'a great deal'. For the purposes of the present investigation, the 12 items described above were used to create a summary measure of distress by summing the scores for each item dividing by the number of items to produce an overall mean score. The scale was found to have good reliability $(\alpha=0.83)$. The mean score for the present sample was 1.90 (s.d.=0.75; range 1-4.42). 


\section{Statistical analyses}

The associations between the measures of (a) earthquake impact, (b) PTSD symptoms, (c) peri-traumatic stress and (d) disruption distress were modelled via latent variable SEM. The fitted model is shown in Figure 1. This model assumes: (i) earthquake impact was an exogenous variable exerting influence on peri-traumatic stress, disruption distress and PTSD symptoms; (ii) peri-traumatic stress and disruption distress were correlated intervening factors that were influenced by earthquake impact; and (iii) PTSD symptoms were influenced by earthquake impact both directly and indirectly via peri-traumatic stress and disruption distress.

In Figure 1, PTSD1, PTSD2, TS1, TS2, DD1, DD2, and EI1 and EI2 are assumed to be a set of observed indicators of underlying latent constructs representing the severity of PTSD, peri-traumatic stress, disruption distress and earthquake impact respectively. For the purposes of the present analysis, the observed indicators PTSD1, PTSD2, TS1, TS2, DD1, DD2, EI1 and EI2 were constructed using split-half score measures of PTSD symptoms, peri-traumatic stress, disruption distress and earthquake impact scores during the period following the earthquakes. These indicators are assumed to be linked to the underlying latent constructs via a so-called 'true score' model $^{29}$ in which the variance in the observed indicators can be partitioned into true score variance reflecting: (a) the individual's underlying level of PTSD, peri-traumatic stress, disruption distress and earthquake impact; and (b) random error due to unreliability in the observed indicators. The use of split-half indicators reduces the influence of measurement error, as compared with SEM with observed measures. ${ }^{29}$ The correlation matrix for the split-half indicators is presented in Table DS1 in the Data supplement.

The model in Figure 1 was fitted to the variance-covariance matrix of the split-half indicators of the latent variables of PTSD, peri-traumatic stress, disruption distress and earthquake impact using Mplus $^{30}$ and methods of maximum likelihood estimation that were robust to non-normality. Model goodness-of-fit was assessed on the basis of the model chi squared, the root mean square error of approximation (RMSEA) and the comparative fit index (CFI). In well-fitting models, the RMSEA should be less than 0.05 and the CFI close to $1 .^{30}$

In addition, from the fitted model it was possible to estimate the components (i.e. fraction) of the correlation between earthquake exposure and PTSD symptoms that were accounted for by: (a) the direct path from earthquake impact to PTSD symptoms; and (b) each of the indirect pathways via peri-traumatic stress and disruption distress. ${ }^{30}$

To examine whether the final fitted model was robust to the potential influence of covariate factors, the model was then extended to include a set of observed covariate factors drawn from the study database (mental health and substance use disorders during the period 25-30 years; PTSD during the period 25-30 years; family living standards at age 30; gender; New Zealand Maori ethnicity; IQ as measured at ages 8-9; covariate factors are described in the Data supplement) that were significantly $(P<0.05)$ correlated with peri-traumatic stress, disruption distress and PTSD symptoms. No covariate factors were found to be significantly correlated with earthquake impact score. For the structural model, the covariate factors were permitted to

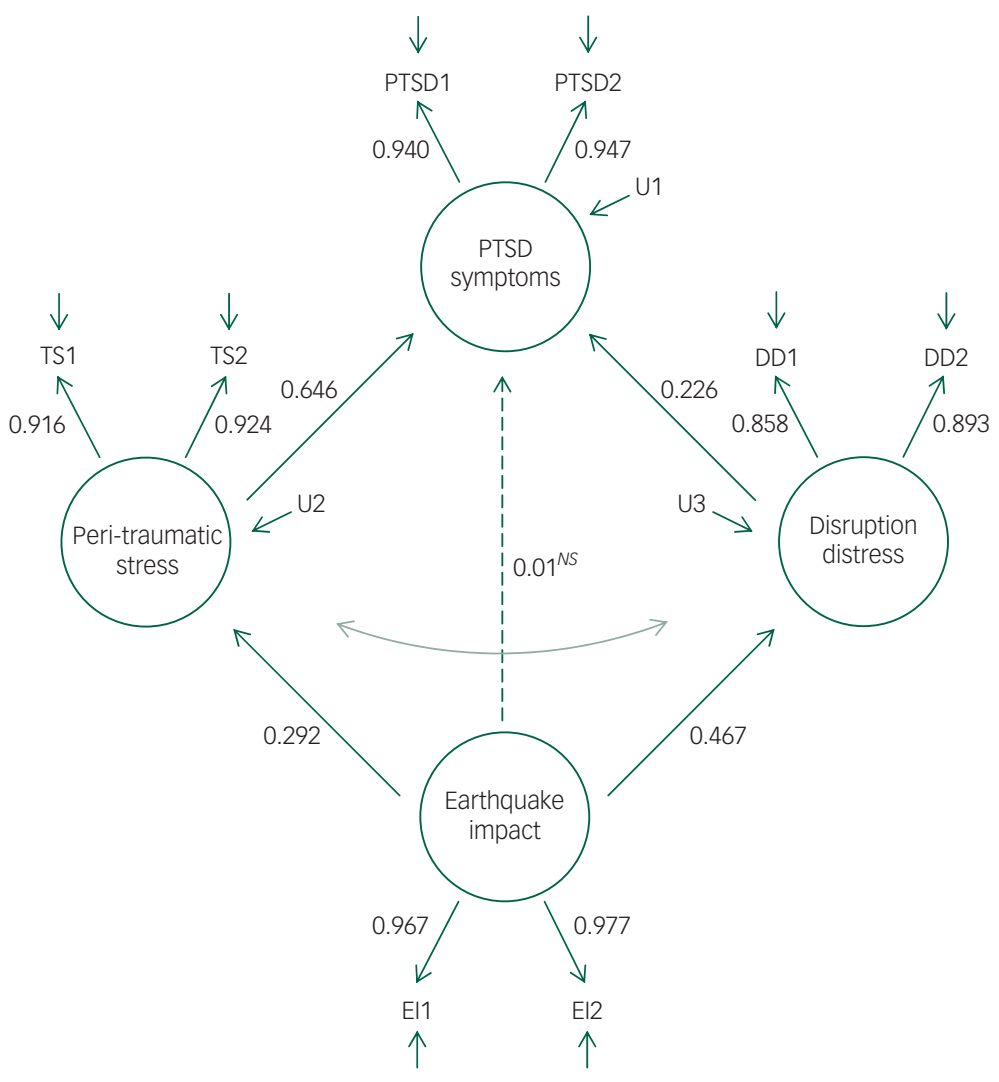

Fig. 1 Fitted structural equation model of the latent measures of earthquake impact score, disruption distress, peri-traumatic stress and post-traumatic stress disorder (PTSD) symptoms, with standardised coefficients.

Note: All paths statistically significant $(P<0.0001)$ unless indicated by ${ }^{N S}$. Indirect path from earthquake impact to PTSD via peri-traumatic stress: $\beta=0.189, S E=0.031, P<0.0001$. Indirect path from earthquake impact to PTSD via disruption distress: $\beta=0.105, \mathrm{SE}=0.025, P<0.0001$ 
be correlated, and peri-traumatic stress, disruption distress and PTSD symptoms were regressed onto the set of covariate factors.

Finally, to examine the robustness of the findings to alternative conceptualisations of the PTSD measure, supplementary analyses were conducted in which the latent measure of PTSD symptoms was replaced by an observed classification of participants of the extent to which they met criteria for a diagnosis of PTSD (see above and Data supplement), and the analyses repeated.

\section{Results}

\section{Structural model of the associations between earthquake impact score and measures of PTSD symptoms}

The SEM depicted in Figure 1 was fitted to the variancecovariance matrix of the split-half indicators of the latent measures of PTSD symptoms, peri-traumatic stress, disruption distress and earthquake impact (see Method and Fig. 1). This model assumed both a direct path from earthquake impact to PTSD symptoms as well as indirect paths via disruption distress and peri-traumatic stress. The model showed an excellent fit to the observed data $\left(\chi^{2}(15)=17.99, \quad P=0.26 ; \quad\right.$ RMSEA $=0.02$; $\mathrm{CFI}=0.999$ ).

Table 1 shows the matrix of correlations between the latent measures of PTSD symptoms, peri-traumatic stress, disruption distress and earthquake impact implied by the fitted model. The table shows that when measurement unreliability was taken into account, there were strong and statistically significant correlations between all four latent measures, with correlation coefficients ranging from 0.292 to 0.792 .

\section{Fitted model results}

The key results of model fitting are displayed in Fig. 1 which shows the standardised regression coefficients for each path in the model, and tests of the indirect pathways from earthquake impact to PTSD symptoms via peri-traumatic stress and disruption distress. Inspection of the figure shows:

1. There were statistically significant $(P<0.0001)$ pathways from earthquake impact to both peri-traumatic stress $(\beta=0.292)$ and disruption distress $(\beta=0.467)$.

2. There were also statistically significant $(P<0.0001)$ pathways to PTSD symptoms from peri-traumatic stress $(\beta=0.646)$ and disruption distress $(\beta=0.226)$.

3. As a consequence of the above pathways, there were statistically significant indirect pathways from earthquake impact to PTSD symptoms via peri-traumatic stress $(\beta=0.189, P<0.0001)$ and via disruption distress $(\beta=0.105$, $P<0.0001)$.

4. After accounting for indirect pathways, the direct effect of earthquake impact on PTSD symptoms was both statistically non-significant and close to zero $(\beta=0.010, P=0.78)$.
In addition, there was a statistically significant correlation between peri-traumatic stress and disruption distress in the fitted model $(r=0.57, P<0.0001)$.

The above results suggest that nearly all of the correlation between earthquake impact and PTSD symptoms were mediated via peri-traumatic stress and disruption distress. The results of the fitted model were used to estimate that component of the correlation between earthquake impact and PTSD symptoms that was accounted for by each of the indirect pathways. Specifically, of the total correlation of 0.304 between earthquake impact and PTSD symptoms, $62.2 \%$ (0.189) was mediated via the indirect pathway through peri-traumatic stress, and 34.5\% (0.105) by the indirect pathway through disruption distress.

\section{Accounting for possible covariate factors}

As noted in the Method, the model was then extended to include a series of covariate factors which were significantly $(P<0.05)$ correlated with peri-traumatic distress, disruption distress and PTSD symptoms. These covariate factors included: mental health and substance use disorders (ages 25-30), PTSD (ages 25-30), family living standards at age 30 , gender, New Zealand Maori ethnicity and IQ as measured at ages 8-9. The results of these analyses showed that the model parameters and goodness-of-fit indices were not materially affected by the inclusion of these covariate factors, suggesting that the linkages between earthquake exposure, peri-traumatic stress, disruption distress and PTSD symptoms were best represented by the more parsimonious model presented in Fig. 1.

\section{Supplementary analysis}

To examine the robustness of the findings to using an alternative measure of PTSD, the above analysis was repeated using the observed three-level diagnostic classification of PTSD (see above) in place of the latent measure of PTSD symptoms. The model showed an excellent fit to the observed data $\left(\chi^{2}(10)=7.90, P=0.64\right.$; RMSEA $=0.00$; CFI $=1.0$ ).

The results of this analysis were very similar to those reported above, with statistically significant $(P<0.0001)$ pathways from earthquake impact to PTSD via peri-traumatic stress and disruption distress, and a statistically non-significant $(P=0.931)$ direct pathway from earthquake impact to PTSD. Of the total correlation of 0.241 between earthquake impact and PTSD, 61.4\% (0.148) was mediated via peri-traumatic stress and $32.4 \%(0.078)$ via disruption distress. The full results of model fitting are provided in Fig. DS1 in the Data supplement.

\section{Discussion}

The present study examined PTSD symptoms, earthquake impact, disruption distress and peri-traumatic stress in response to a major disaster, the Canterbury earthquakes of 2010-2011, among members of a well-studied birth cohort, using SEM to examine the linkages between these factors. This analysis led to several general conclusions.

Table 1 Correlation matrix for latent measures of earthquake impact score, disruption distress, peri-traumatic stress and post-traumatic

\begin{tabular}{|c|c|c|c|c|}
\hline Measure & Earthquake impact & Disruption distress & Traumatic stress & PTSD symptoms \\
\hline Earthquake impact & 1.0 & 0.467 & 0.292 & 0.304 \\
\hline Disruption distress & & 1.0 & 0.633 & 0.639 \\
\hline Peri-traumatic stress & & & 1.0 & 0.792 \\
\hline PTSD symptoms & & & & 1.0 \\
\hline
\end{tabular}


The analysis using SEM showed that the linkages between earthquake impact and earthquake-related PTSD symptoms were explained by two factors: first, by the experience of peri-traumatic stress during the earthquakes; and second, via disruption distress. The fitted structural model showed that the direct influence of earthquake impact on PTSD symptomatology was almost nil. The fraction of the correlation between earthquake impact and PTSD symptoms that was attributable to the indirect pathway via peri-traumatic stress was $62.2 \%$, and the fraction of the correlation between earthquake impact and PTSD symptoms accounted for by the indirect pathway via disruption distress was $34.5 \%$. Furthermore, a similar pattern of results was found using a measure in which participants were classified in terms of meeting criteria for a diagnosis of PTSD, suggesting that the models were robust to alternative conceptualisations of earthquake-related PTSD symptoms.

The results of the present study are in agreement with a range of studies that show that exposure to potentially life-threatening events during a natural disaster is linked to increased risk of PTSD symptomatology. ${ }^{8-11}$ At the same time, the results also suggest that the key determinants of PTSD symptomatology in response to a disaster are not only the extent to which the individual experienced intense fear/peri-traumatic stress during the event, but also the extent to which there is distress over lingering disruption following the event. These findings are consistent with evidence suggesting that the experience of PTSD is strongly linked to the neural circuitry involved in fear responses ${ }^{15-17}$ and evidence showing that lingering disruptive effects of disaster exposure are also related to PTSD symptomatology. ${ }^{18-20}$

These findings may have key implications for research examining the mental health impacts of disaster exposure. A number of studies with varying methodologies have found generally weak to moderate effects of disaster exposure on mental health, ${ }^{3,5,7,31,32}$ with similar findings being reported in an earlier study of the present cohort. ${ }^{24}$ The findings of the present analysis suggest that one of the reasons for the relatively modest associations between disaster exposure and mental health disorders may be individual differences in fear/peri-traumatic stress responses during the disaster event. Evidence from animal models suggests that there are individual differences in fear and stress responses to the same aversive stimulus. ${ }^{33}$ The present data suggest that an individual's emotional response to events both during and following the disaster may play a critical role in determining whether the individual develops PTSD symptoms following disaster exposure.

These findings also highlight the importance of studies of natural disasters obtaining reports of a range of facets related to the disorder, in addition to mental health and related measures. The present findings suggest that an understanding of the multifaceted nature of disaster exposure is critical in assessing mental health risk and service needs in the aftermath of a natural disaster. ${ }^{12,13}$

A further issue raised by the present study is that approaches to identifying and treating individuals with PTSD following disaster exposure must consider the fact that these symptoms may continue for a long period following exposure, particularly when the disruption related to the disaster is not alleviated in a timely manner. In the present study, the assessment of symptoms took place up to 24 months after exposure to the earthquakes, suggesting that there were increased mental health needs among those exposed, over a long period of time. Earlier research has also suggested that PTSD may persist for a number of years following disaster exposure, ${ }^{34-36}$ and further research is needed to determine whether these longer-term adverse mental health outcomes may be alleviated by timely and focused mental health interventions for those exposed. ${ }^{37}$

The findings of the present study may also have implications for changes to the PTSD diagnostic criteria that appeared in DSM-5. ${ }^{38,39}$ In DSM-5, the former criterion 'A2' relating to subjective response to the traumatic event was removed. The results of the present study imply that this change may have been in error, and that it may be important to retain consideration of subjective response to the event in the peri- and post-traumatic periods.

Although the present study has a number of advantages, primarily due to the availability of data from a well-studied cohort, this feature is also a limitation of the study since the findings are limited to populations in their mid-30s. It is possible that reactions to the Canterbury earthquakes varied with age, with the result that reactions among younger and older populations may be different from those found for this cohort.

A further limitation may be the time frame for assessment, which was 20-24 months after the onset of the earthquake sequence, limits the conclusions to longer-term reactions to earthquake exposure. It may be possible, for example, that peritraumatic stress is more strongly predictive of shorter-term PTSD symptoms than disruption distress, whereas the opposite may be true for longer-term PTSD symptoms. Future assessment and analyses of the CHDS cohort will allow these questions to be addressed.

An additional limitation is that the assessment of earthquake exposure may be limited by the extent to which self-reported personal perceptions of the events may be subject to the usual biases inherent in self-report.

In addition, it should be noted that only a small minority (3.2\%) of respondents met criteria for a diagnosis of PTSD, which implies that the parameter estimates obtained in the supplementary analysis using the three-level measure of PTSD outcomes may be somewhat less reliable than the primary model employing a PTSD symptom count.

Finally, the present data are unable to address questions as to whether the increased level of symptoms related to disruption distress reflect adjustment disorder rather than PTSD per se. Research suggests that differentiation between the two disorders is not a straightforward issue, ${ }^{40}$ and further research is necessary in this area.

\footnotetext{
Joseph M. Boden, PhD, Department of Psychological Medicine, University of Otago, Christchurch School of Medicine and Health Sciences, Christchurch, New Zealand; David M. Fergusson, PhD, Department of Psychological Medicine, University of Otago, Christchurch School of Medicine and Health Sciences, Christchurch, New Zealand; L. John Horwood, MSC, Department of Psychological Medicine, University of Otago, Christchurch School of Medicine and Health Sciences, Christchurch, New Zealand; Roger T. Mulder, MB, ChB, PhD, Department of Psychological Medicine, University of Otago, Christchurch School of Medicine and Health Sciences, Christchurch, New Zealand

Correspondence: Joseph M. Boden, PhD, Associate Professor, Christchurch Health and Development Study, University of Otago, Christchurch School of Medicine and Health Sciences, PO Box 4345, Christchurch, New Zealand. Email: joseph.boden@otago. ac.nz

First received 11 Jun 2015, final revision 7 Jul 2015, accepted 30 Jul 2015
}

\section{Funding}

This research was funded by grants from the Health Research Council of New Zealand, the National Child Health Research Foundation, the Canterbury Medical Research Foundation and the New Zealand Lottery Grants Board. 


\section{References}

1 Green BL. Psychological responses to disasters: conceptualization and identification of high-risk survivors. Psychiatry Clin Neurosci 1998; 52: S25-31.

2 Kar N. Psychological impact of disasters on children: review of assessment and interventions. World J Pediatr 2009; 5: 5-11.

3 Norris FH, Friedman MJ, Watson PJ, Byrne CM, Diaz E, Kaniasty K. 60,000 Disaste victims speak: part I. An empirical review of the empirical literature, 1981-2001. Psychiatry 2002; 65: 207-39.

4 Schnurr PP, Green BL. Understanding relationships among trauma, post-traumatic stress disorder, and health outcomes. Adv Mind Body Med 2004; 20: 18-29.

5 Rubonis AV, Bickman L. Psychological impairment in the wake of disaster: the disaster-psychopathology relationship. Psychol Bull 1991; 109: 384-99.

6 Flannery RB, Jr. Psychological trauma and posttraumatic stress disorder: a review. Int J Emerg Ment Health 1999; 1: 135-40.

7 Foa EB, Stein DJ, McFarlane AC. Symptomatology and psychopathology of menta health problems after disaster. J Clin Psychiatry 2006; 67 (suppl 2): 15-25.

8 Aker AT. 1999 Marmara earthquakes: a review of epidemiologic findings and community mental health policies. Türk Psikiyatri Dergisi 2006; 17: 204-12.

9 Amstadter AB, Acierno R, Richardson LK, Kilpatrick DG, Gros DF, Gaboury MT, et al. Posttyphoon prevalence of posttraumatic stress disorder, major depressive disorder, panic disorder, and generalized anxiety disorder in a Vietnamese sample. J Trauma Stress 2009; 22: 180-8.

10 Yang $\mathrm{P}$, Yen CF, Tang TC, Chen CS, Yang RC, Huang MS, et al. Posttraumatic stress disorder in adolescents after Typhoon Morakot-associated mudslides. J Anxiety Disord 2011; 25: 362-8

11 Van Griensven F, Chakkraband MLS, Thienkrua W, Pengjuntr W, Lopes Cardozo B, Tantipiwatanaskul $P$, et al. Mental health problems among adults in tsunamiaffected areas in southern Thailand. J Am Med Assoc 2006; 296: 537-48.

12 Albala-Bertrand JM. Complex emergencies versus natural disasters: an analytica comparison of causes and effects. Oxford Dev Stud 2000; 28: 187-204.

13 King $\mathrm{E}$, Mutter JC. Violent conflicts and natural disasters: the growing case for cross-disciplinary dialogue. Third World Q 2014; 35: 1239-55.

14 Galea S, Tracy M, Norris F, Coffey SF. Financial and social circumstances and the incidence and course of PTSD in Mississippi during the first two years afte Hurricane Katrina. J Trauma Stress 2008; 21: 357-68.

15 Mahan AL, Ressler KJ. Fear conditioning, synaptic plasticity and the amygdala: implications for posttraumatic stress disorder. Trends NeurosCi 2012; 35: 24-35.

16 Jovanovic T, Ressler KJ. How the neurocircuitry and genetics of fear inhibition may inform our understanding of PTSD. Am J Psychiatry 2010; 167: 648-62.

17 Blechert J, Michael T, Vriends N, Margraf J, Wilhelm FH. Fear conditioning in posttraumatic stress disorder: evidence for delayed extinction of autonomic experiential, and behavioural responses. Behav Res Ther 2007; 45: 2019-33.

18 Newnham EA, Pearson RM, Stein A, Betancourt TS. Youth mental health after civil war: the importance of daily stressors. Br J Psychiatry 2014; 206: 116-21.

19 Parslow RA, Jorm AF, Christensen H. Associations of pre-trauma attributes and trauma exposure with screening positive for PTSD: analysis of a community-based study of 2085 young adults. Psychol Med 2006; 36: 387-95.

20 Tracy $\mathrm{M}$, Norris $\mathrm{FH}$, Galea S. Differences in the determinants of posttraumatic stress disorder and depression after a mass traumatic event. Depress Anxiety 2011; 28: 666-75.
21 Fergusson DM. The Christchurch Health and Development Study: an overview and some key findings. Social Pol J New Zeal 1998; 10: 154-76.

22 Fergusson DM, Horwood $\sqcup$. The Christchurch Health and Development Study: Review of findings on child and adolescent mental health. Aust $N Z J$ Psychiatry 2001; 35: 287-96.

23 Dowrick DJ. The modified Mercalli earthquake intensity scale; revisions arising from recent studies of New Zealand earthquakes. Bull New Zeal Natl SOC Earthquake Eng 1996; 29: 92-106.

24 Fergusson DM, Horwood $\sqcup$, Boden JM, Mulder RT. Impact of a major disaster on the mental health of a well-studied cohort. JAMA Psychiatry 2014; 71: 1025-31.

25 Robins LN, Cottler L, Bucholz K, Compton W. Diagnostic Interview Schedule for DSM-IV. Washington University Press, 1995

26 American Psychiatric Association. Diagnostic and Statistical Manual of Mental Disorders (4th edn) (DSM-IM). APA, 1994.

27 Brunet A, Weiss DS, Metzler TJ, Best SR, Neylan TC, Rogers C, et al. The Peritraumatic Distress Inventory: a proposed measure of PTSD criterion A2. Am J Psychiatry 2001; 158: 1480-5.

28 Fergusson DM, Boden JM, Horwood $\sqcup$, Mulder RT. Perceptions of distress and positive consequences following exposure to a major disaster amongst a well-studied cohort. Aust N Z J Psychiatry 2015; 49: 351-9.

29 Alwin DF, Jackson GJ. Measurement models for response errors in surveys: issues and applications. In Sociological Methodology (ed. KF Schuessler): 69-119. Jossey-Bass, 1980.

30 Muthen LK, Muthen BO. Mplus Users' Guide. Muthen and Muthen, 2007.

31 North CS, Pfefferbaum B. Mental health response to community disasters: a systematic review. JAMA 2013; 310: 507-18.

32 Galea S, Nandi A, Vlahov D. The epidemiology of post-traumatic stress disorder after disasters. Epidemiol Rev 2005; 27: 78-91.

33 Liu J, Wei W, Kuang H, Tsien J, Zhao F. Heart rate and heart rate variability assessment identifies individual differences in fear response magnitudes to earthquake, free fall, and air puff in mice. PLoS One 2014; 9: e93270.

34 Yule W, Bolton D, Udwin O, Boyle S, O'Ryan D, Nurrish J. The long-term psychological effects of a disaster experienced in adolescence: I: the incidence and course of PTSD. J Child PSychol Psychiatry 2000; 41: 503-11.

35 Green BL, Lindy JD, Grace MC, Leonard AC. Chronic Posttraumatic-stress-disorder and diagnostic comorbidity in a disaster sample. J Nerv Ment Dis 1992; 180 $760-6$.

36 DiGrande L, Neria Y, Brackbill RM, Pulliam P, Galea S. Long-term posttraumatic stress symptoms among 3,271 civilian survivors of the September 11, 2001, terrorist attacks on the World Trade Center. Am J Epidemiol 2010; 173: 271-81.

37 Kearns MC, Ressler KJ, Zatzick D, Olasov Rothbaum B. Early interventions for PTSD: a review. Depress Anxiety 2012; 29: 833-42.

38 American Psychiatric Association. Diagnostic and Statistical Manual of Mental Disorders, 5th Edition (DSM-5). APA, 2013.

39 O'Donnell ML, Alkemade N, Nickerson A, Creamer M, McFarlane AC, Silove D, et al. Impact of the diagnostic changes to post-traumatic stress disorder for DSM-5 and the proposed changes to ICD-11. Br J Psychiatry 2014; 205: 230-5.

40 Casey P, Bailey S. Adjustment disorders: the state of the art. World Psychiatry 2011: 10: 11-8. 